\title{
New pathogenetic insights into Charcot-Marie-Tooth disease
}

Researchers in Belgium have generated a mouse model of Charcot-Marie-Tooth disease (CMT), and have discovered that axonal transport defects are associated with disease pathology. The study, published in Nature Medicine, highlights a potential mechanism to restore axonal transport and reverse peripheral neuropathy.

Previous studies have shown that the gene encoding heat shock protein $\mathrm{B} 1$ (HSPB1) is mutated in cases of CMT2F, an axonal form of CMT. "We aimed to assess whether the expression of mutant human HSPB1 in neurons was necessary and sufficient to cause a phenotype resembling CMT," explains lead researcher Ludo Van Den Bosch.

Transgenic mice that overexpressed mutant HSPB1 developed normally, but started to show progressive symptoms of motor defects, sensory impairments, muscle atrophy and weakness, and limb deformities from 6 months of age. As in patients with CMT, contacts between nerve endings and muscles were disturbed in these mice.

Defective axonal transport has been identified as an underlying cause of pathology in motor neuron diseases.
"Mitochondrial transport was severely disturbed in neurons isolated from symptomatic mutant HSPB1-expressing mice," says Van Den Bosch.

Mitochondria are transported along microtubules, which consist of polymerized tubulin. "We were particularly interested in the acetylation of a-tubulin, because this process is a major cue for anchoring motor proteins to microtubules," explains Van Den Bosch. Levels of acetylated tubulin were significantly reduced in both cultured neurons and neuronal tissue homogenate from transgenic mice, compared with tissue from healthy control mice.

\section{These findings indicate that HDAC6 has a crucial role in the pathogenesis of CMT 77}

Treatment with tubastatin A, a small-molecule inhibitor of the tubulin deacetylating enzyme histone deacetylase 6 (HDAC6), increased the levels of acetylated tubulin, restored axonal transport defects, reversed axonal loss, and rescued the behavioral phenotype of mice expressing mutant HSPB1. These findings indicate that HDAC6 has a crucial role in the pathogenesis of CMT.

"Our studies show that restricted overexpression of mutant HSPB1 in neurons is sufficient to induce a CMTlike phenotype in these animals," states Van Den Bosch. His team is now investigating the mechanisms through which the HSPB1 mutation causes HDAC6-mediated deacetylation of tubulin, as well as exploring a potential role for tubulin deacetylation in other neurological disorders.

In the future, it might be possible to produce inhibitors of tubulin deacetylation that have beneficial effects in individuals with CMT. As Van Den Bosch points out, however, there is still a long way to go before this goal is achieved. Meanwhile, he hopes that the transgenic model developed by his research team can help in the search for a cure for CMT and other peripheral neuropathies.

Katy Malpass

Original article d'Ydewalle, C. et al. HDAC6 inhibitors reverse axonal loss in a mouse model of mutant HSPB1induced Charcot-Marie-Tooth disease. Nat. Med. 17, 968-974 (2011) 